\title{
Higher-Order Corrections to Algebraic Derivation of Electric Dipole-Dipole Interaction
}

\author{
Haiduke Sarafian \\ The Pennsylvania State University, University College, York, USA \\ Email: has2@psu.edu
}

How to cite this paper: Sarafian, H. (2021) Higher-Order Corrections to Algebraic Derivation of Electric Dipole-Dipole Interaction. American Journal of Computational Mathematics, 11, 267-272.

https://doi.org/10.4236/ajcm.2021.114017

Received: November 2, 2021

Accepted: December 5, 2021

Published: December 8, 2021

Copyright (c) 2021 by author(s) and Scientific Research Publishing Inc. This work is licensed under the Creative Commons Attribution International License (CC BY 4.0).

http://creativecommons.org/licenses/by/4.0/

\begin{abstract}
Nucleons are fermions with intrinsic spins exhibiting dipole character. Dipole-dipole interaction via their dipole moments is the key feature quantifying the short-range nucleonics interaction in two-body physics. For a pair of interacting dipoles, the energy of a pair is the quantity of interest. The same is true for chemical polar molecules. For both cases, derivation of energy almost exclusively is carried out vectorially [1]. Although uncommon the interacting energy can be derived algebraically too. For the latter Taylor, expansion is applied [2]. The given expression although appears to be correct it is incomplete. In our report, by applying Taylor's expansion up to the 4th order and utilizing a Computer Algebra System we formulate the missing terms. Our report highlights the impact of correcting missing terms by giving two explicit examples.
\end{abstract}

\section{Keywords}

Electric Dipole-Dipole Interaction, Taylor Expansion, Computer Algebra System (CAS), Mathematica

\section{Introduction}

Reviewing literature on the format of dipole-dipole interaction energy in three areas of physics, magnetic, nuclear, and electric shows the similarity of the formulations. Denoting dipole moments by, $\vec{\mu}, \vec{\sigma}$, and $\vec{m}$, respectfully, these are,

$$
\begin{gathered}
U\left(\vec{\mu}_{1}, \vec{\mu}_{2}\right)=C_{1} \frac{1}{r^{3}}\left[\vec{\mu}_{1} \cdot \vec{\mu}_{2}-3 \vec{\mu}_{1} \cdot \hat{r} \vec{\mu}_{2} \cdot \hat{r}\right], \\
U\left(\vec{\sigma}_{1}, \vec{\sigma}_{2}\right)=C_{2} \frac{1}{r^{3}}\left[\vec{\sigma}_{1} \cdot \vec{\sigma}_{2}-3 \vec{\sigma}_{1} \cdot \hat{r} \vec{\sigma}_{2} \cdot \hat{r}\right],
\end{gathered}
$$




$$
U\left(\vec{m}_{1}, \vec{m}_{2}\right)=C_{3} \frac{1}{r^{3}}\left[\vec{m}_{1} \cdot \vec{m}_{2}-3 \vec{m}_{1} \cdot \hat{r} \vec{m}_{2} \cdot \hat{r}\right]
$$

The constant coefficients $\mathcal{C}$ s are associated with the coupling constants of each case. In these expressions, $r$ is the vector distance between the dipoles with $\hat{r}$ being its associated unit vector.

\section{Calculation}

These expressions have been derived applying vector analysis e.g. [3]. For instance, the derivation of (1) is based on the gained energy of a magnet with a magnetic dipole $\vec{\mu}_{2}$ in the magnetic field of another magnet with a dipole moment $\vec{\mu}_{1}$.

Motivated with [2] we revisited its algebraic derivation. We consider a pair of electric dipoles in a 2D space. Figure 1 describes the scenario.

The $\pm q$ charges of each dipole are separated by a distance of $2 d$. For the sake of calculation, midpoints are used indicating the separation of the dipoles $\vec{r}_{12}$. As shown, there are four paired charges; these are labeled with their connecting-colored dashed lines; $1,2,3$, and 4 . These lines are indicative of repulsive and/or attractive interactions. The blue dashed lines 1,2 join the $+q$ to $-q$ of individual dipole while the red dashed lines 3, 4 join the corresponding $+q$ 's and $-q$ s. Aside from the electrostatic coupling constant $k=\frac{1}{4 \pi \varepsilon_{0}}=8.95 \times 10^{9} \frac{\mathrm{N} \cdot \mathrm{m}^{2}}{\mathrm{C}^{2}}$ and the charge $\mathrm{q}$ the electrostatic potential energy of the paired dipoles according to Figure 1 is, $U=\sum_{i=1}^{\prime 4} \pm \frac{1}{\text { dist }_{i}}$ where $\Sigma^{\prime}$ is the algebraic sum, and dist $_{i}$ is the distance between the pairs, namely $i=1,2,3$ and 4. Applying this notation according to vectors shown in Figure 1 the potential energy reads,

$$
U=-\left[\frac{1}{\left|\vec{r}+\left(\vec{d}_{2}-\vec{d}_{1}\right)\right|}+\frac{1}{\left|\vec{r}+\left(-\vec{d}_{2}+\vec{d}_{1}\right)\right|}\right]+\left[\frac{1}{\left|\vec{r}+\left(\vec{d}_{2}+\vec{d}_{1}\right)\right|}+\frac{1}{\left|\vec{r}-\left(\vec{d}_{2}+\vec{d}_{1}\right)\right|}\right]
$$

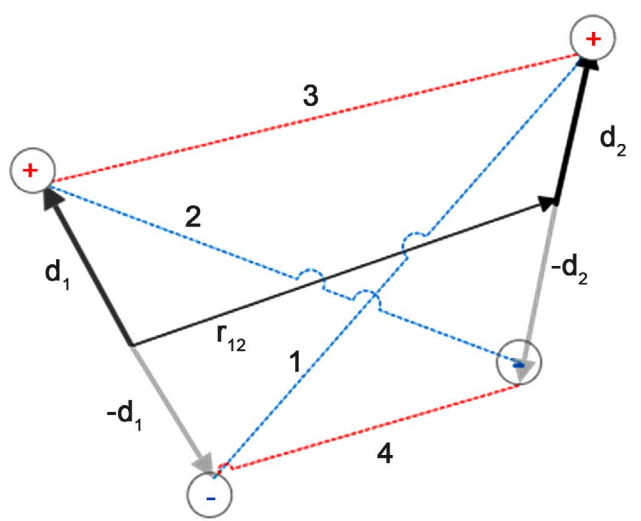

Figure 1. Two electric dipoles with identical dipole moments $2 d q$ are oriented as shown. Vector $\vec{r}_{12}$ connects their centers. 
As noted, for the sake of simplicity in (4), $\vec{r}_{12}$ is replaced with $\vec{r}$. The first two terms and the last two terms in (4) correspond to lines 1, 2 and 3, 4, shown in Figure 1, respectively. Note also according to notation: $\vec{d}_{1}=\frac{d}{2} \hat{d}_{1}$ and $\vec{d}_{2}=\frac{d}{2} \hat{d}_{2}$. These are conducive to dipole moments, $\vec{m}_{1}=q d \hat{d}_{1}$ and $\vec{m}_{2}=q d \hat{d}_{2}$.

One notices all four terms in (4) are common in $1 / r$ and since we are confined in a $2 \mathrm{D}$ space this means $\frac{1}{\sqrt{x^{2}+y^{2}}}$. The objective of the calculation is to apply Taylor expansion, as such the latter fraction is expanded for each of the four terms of (4) according to their increments. Let's define

$$
f(x, y):=\frac{1}{\sqrt{x^{2}+y^{2}}}
$$

Expanding the first term of (4) with its associated increment gives,

$$
\begin{aligned}
& f\left(x+\left(d_{2}-d_{1}\right)_{x}, y+\left(d_{2}-d_{1}\right)_{y}\right) \\
& =f(x, y)+\left[\partial_{x} f(x, y)\left(d_{2}-d_{1}\right)_{x}+\partial_{y} f(x, y)\left(d_{2}-d_{1}\right)_{y}\right] \\
& +\frac{1}{2 !}\left[\partial_{x}^{2} f(x, y)\left(d_{2}-d_{1}\right)_{x}^{2}+2 \partial_{x, y} f(x, y)\left(d_{2}-d_{1}\right)_{x}\left(d_{2}-d_{1}\right)_{y}\right. \\
& \left.+\partial_{y}^{2} f(x, y)\left(d_{2}-d_{1}\right)_{y}^{2}\right]+\frac{1}{3 !} 0^{3}+\frac{1}{4 !}\left[\partial_{x}^{4} f(x, y)\left(d_{2}-d_{1}\right)_{x}^{4}\right. \\
& +4 \partial_{x}^{3} \partial_{y} f(x, y)\left(d_{2}-d_{1}\right)_{x}^{3}\left(d_{2}-d_{1}\right)_{y}+6 \partial_{x}^{2} \partial_{y}^{2} f(x, y)\left(d_{2}-d_{1}\right)_{x}^{2}\left(d_{2}-d_{1}\right)_{y}^{2} \\
& \left.+4 \partial_{x} \partial_{y}^{3} f(x, y)\left(d_{2}-d_{1}\right)_{x}\left(d_{2}-d_{1}\right)_{y}^{3}+\partial_{y}^{4} f(x, y)\left(d_{2}-d_{1}\right)_{y}^{4}\right]+\cdots
\end{aligned}
$$

In (6), $\mathrm{O}^{3}$ is the $3^{\text {rd }}$ order term, its format is like the $2^{\text {nd }}$ and the $4^{\text {th }}$ term. Because of its collective null value, it is not given explicitly. Noting also $\partial_{x}^{4}$ means $\partial_{x, x, x, x}$, etc. Equation (6) must be applied to the remaining three terms of (4). Realizing the first two terms of (4) are negative and the last two are positive yields to the cancelation of the first term of (6) when applied to (4). The second term of (6) contains first-order partial derivatives. Increments of four terms of (4) are such that when the second term of (6) is applied to (4) gives zero. Further analysis shows in general all odd orders of (6) when applied to (4) yield zero. In other words, only even terms of (6) survive. i.e., Taylor expansion of (4) may replace (4) in terms of only even powered terms.

As such we calculate the first even powered term of Taylor expansion, this is,

$$
\begin{aligned}
& \left\{\partial_{x, x} f[x, y], 2 \partial_{x, y} f[x, y], \partial_{y, y} f[x, y]\right\} \\
& =\left\{\frac{3 x^{2}}{\left(x^{2}+y^{2}\right)^{5 / 2}}-\frac{1}{\left(x^{2}+y^{2}\right)^{3 / 2}}, \frac{6 x y}{\left(x^{2}+y^{2}\right)^{5 / 2}}, \frac{3 y^{2}}{\left(x^{2}+y^{2}\right)^{5 / 2}}-\frac{1}{\left(x^{2}+y^{2}\right)^{3 / 2}}\right\}
\end{aligned}
$$

Equation (7) reveals the functional format of the expression. Simply put denominators are of form $1 / r^{3}$ and $1 / r^{5}$, these are compatible with the functional formats of (1)-(3). Now each term of (7) needs to be multiplied by their asso- 
ciated increments. Carrying out this algebraic simplification is cumbersome, that is the reason it is carried out applying a CAS, specifically Mathematica [4]. Nonetheless, its simplified output needs to be format-ed using the defined $\vec{m}_{1}$ and $\vec{m}_{2}$ in the text. This step requires regrouping of terms and is handled by hand. The result is identical to (3).

Successfully this algebraic procedure bypasses the vector analysis producing the standard energy associated with the dipole-dipole interaction. However, Taylor expansion should not be terminated to the $2^{\text {nd }}$ order! As mentioned earlier higher even order terms should be included. It is one of the objectives of this report to investigate the impact of the next, i.e., the $4^{\text {th }}$. One may argue contribution of terms beyond the $2^{\text {nd }}$ order "should" be ignored. Reasoning these terms would be falling off vs. distance as $1 / r^{7}$ and $1 / r^{9}$. Of course, the argument holds so long as separation distance is large, however, for short distances this is not true. Even more interesting, here we show the $4^{\text {th }}$ order term contains a term such as $1 / r^{5}$, and that needs to be added to the term of the same format that comes about from the $2^{\text {nd }}$ order Taylor expansion. Here is the proof. Noting the $4^{\text {th }}$ order term of the Taylor expansion as pointed out in (6) contains $4^{\text {th }}$ order partial derivatives we form its derivatives,

$$
\begin{aligned}
\{ & \left.\partial_{x, x, x, x} f[x, y], 4 \partial_{x, x, x, y} f[x, y], 6 \partial_{x, x, y, y} f[x, y], 4 \partial_{x, y, y, y} f[x, y], \partial_{y, y, y, y} f[x, y]\right\} \\
= & \left\{\frac{105 x^{4}}{\left(x^{2}+y^{2}\right)^{9 / 2}}-\frac{90 x^{2}}{\left(x^{2}+y^{2}\right)^{7 / 2}}+\frac{9}{\left(x^{2}+y^{2}\right)^{5 / 2}}, 4\left(\frac{105 x^{3} y}{\left(x^{2}+y^{2}\right)^{9 / 2}}-\frac{45 x y}{\left(x^{2}+y^{2}\right)^{7 / 2}}\right),\right. \\
& 6\left(\frac{105 x^{2} y^{2}}{\left(x^{2}+y^{2}\right)^{9 / 2}}-\frac{15 x^{2}}{\left(x^{2}+y^{2}\right)^{7 / 2}}-\frac{15 y^{2}}{\left(x^{2}+y^{2}\right)^{7 / 2}}+\frac{3}{\left(x^{2}+y^{2}\right)^{5 / 2}}\right), \\
& \left.4\left(\frac{105 x y^{3}}{\left(x^{2}+y^{2}\right)^{9 / 2}}-\frac{45 x y}{\left(x^{2}+y^{2}\right)^{7 / 2}}\right), \frac{105 y^{4}}{\left(x^{2}+y^{2}\right)^{9 / 2}}-\frac{90 y^{2}}{\left(x^{2}+y^{2}\right)^{7 / 2}}+\frac{9}{\left(x^{2}+y^{2}\right)^{5 / 2}}\right\}
\end{aligned}
$$

If in (8) virtually we replace $\sqrt{x^{2}+y^{2}}$ with $r$, we see that there are three terms in proportion to $1 / r^{5}$; these cannot be ignored and ought to be added to the terms of the same format when the $2^{\text {nd }}$ order expansion is considered. The other terms in (8) using the $r$ notation are of the form $1 / r^{7}$ and $1 / r^{9}$; depending on the situation these terms may be ignored. It needs to be noted that it is obvious that higher-order expansion terms appear to contain overlapping $1 / r^{n}$ terms of the lower order. E.g., a sixth-order Taylor expansion would contain a term such as $1 / r^{7}$ and that needs to be added to the $4^{\text {th }}$ order, etc.

Now as it is done for the $2^{\text {nd }}$ order, terms of the (8) are to be multiplied with their associated increments and then to be factored with $1 / r^{5}$. This is indeed a very labor-intensive algebraic procedure. This is carried out applying CAS, Mathematica. The output is an algebraic expression with 44 terms! The terms are regrouped long-hand. In the end, utilizing the dipole moment notations we arrive at, 


$$
U\left(\vec{m}_{1}, \vec{m}_{2}\right)=C_{3}\left\{\frac{\vec{m}_{1} \cdot \vec{m}_{2}}{r^{3}}-\frac{3}{r^{5}}\left[\vec{m}_{1} \cdot \vec{r} \vec{m}_{2} \cdot \vec{r}-\frac{1}{8} \vec{m}_{1} \cdot \vec{m}_{2}\left(\vec{m}_{1} \cdot \vec{m}_{1}+\vec{m}_{2} \cdot \vec{m}_{2}\right)\right]\right\}
$$

Equation (9) is compared to (3). The second expression in the angled bracket is the correction term. Its dimension is $\mathrm{L}^{-1}$ as is the dimension of the expression in the curly bracket. The constant $C_{3}$ is $\mathrm{kq}^{2}$ where the $k$ is the electrostatic coupling constant and $\mathrm{q}$ is the charge of the dipole making the dimension (9) energy as expected.

To illustrate the impact of the correction term we consider two instances. Figure 2(a) and Figure 2(b) depict the cases of interest.

The values of the moments may be chosen selectively, for the sake of simplicity here we assume $m_{1}=m_{2}=1$. According to Figure 2(a) and Figure 2(b), (9) without and with correction terms (3) yields, respectfully.

$$
\begin{aligned}
& U \sim\left\{\frac{1}{x^{3}}, \frac{1}{x^{3}}+\frac{3}{4} \frac{1}{x^{5}}\right\} \\
& U \sim\left\{\frac{1}{y^{3}}, \frac{1}{y^{3}}-\frac{9}{4} \frac{1}{y^{5}}\right\}
\end{aligned}
$$

In (10) and (11), $\mathrm{x}$ and $\mathrm{y}$ are the horizontal and vertical distances between the centers of dipoles.

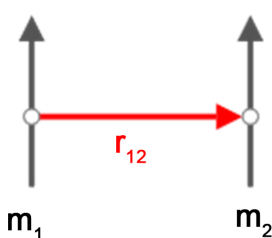

(a)

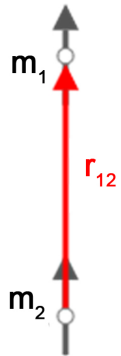

(b)

Figure 2. Two parallel electric dipoles are shown. (a) Both dipoles are perpendicular to the vector $\vec{r}_{12}$ separating the dipoles. (b) Dipoles are aligned with the separating vector $\vec{r}_{12}$.

$$
\mathrm{m}_{1} \| \mathrm{m}_{2} \text { \& both } \perp \text { to } \hat{\mathrm{r}}
$$

\section{Energy}

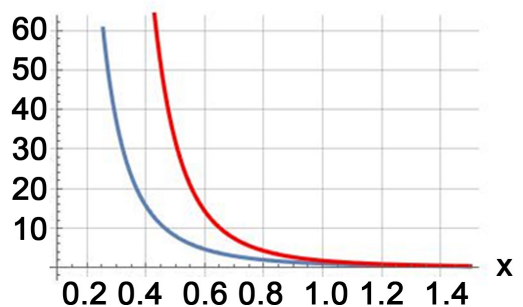

$\mathrm{m}_{1} \| \mathrm{m}_{2}$ \& both $\|$ to $\hat{r}$

\section{Energy}

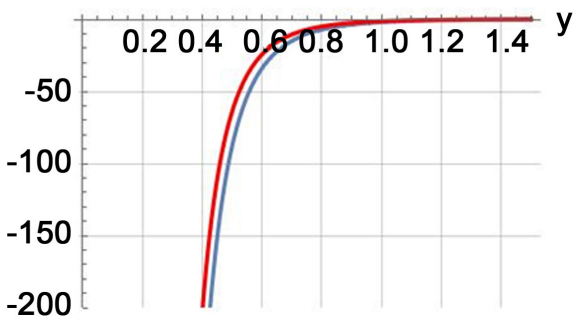

Figure 3. The left panel is associated with Figure 2(a), the right panel is with Figure 2(b). 
In Figure 3, the blue curves are the uncorrected expressions in (10) and (11). The red curves are the corrected expressions. It is notice-able the correction terms have no neglect-able impacts. For the case shown in Figure 2(a), the impact is large, while for Figure 2(b) it is not severe.

We shown two instances to highlight the impact of the corrections. There are other cases correlated with Figure 2, such as antiparallel-oriented dipoles. These and other cases are left to the interested reader to explore.

\section{Conclusion}

Dipole-dipole interaction conducive to either mutual paired forces or energy redundantly is utilized in various areas of physics. Derivation of these quantities exclusively is based on vector calculation. An attempt was made in the referenced article to derive the same expressions applying algebra, specifically Taylor expansion that bypasses the latter. Although it is shown the goal is "achievable", but the calculation falls short by limiting the number of the expansion terms. In our investigation explicitly we have shown higher-order terms beyond the second order ought to be considered. This stresses that although the algebraic approach is novel, it must be done with much care.

\section{Acknowledgements}

The author acknowledges the John T. and Page S. Smith Professorship funds for completing and publishing this work.

\section{Conflicts of Interest}

The author declares no conflicts of interest regarding the publication of this paper.

\section{References}

[1] Parker, G.W. (1985) Derivation of the Electric Dipole-Dipole Interaction as an Electric Hyperfine Interaction. American Journal of Physics, 54, 715-717. https://doi.org/10.1088/0031-9120/45/4/003

[2] Kocback, L. and Lubbad, S. (2009) Geometrical Simplification of the Dipole-Dipole Interaction Formula. Physics Education, 45, 345-351. https://doi.org/10.1088/0031-9120/45/4/003

[3] Low, F. (1997) Classic Field Theory. John Wiley, New York.

[4] Mathematica Version 12.3. http://Wolfram.com 